\title{
Observer independent analysis of cerebral glucose metabolism in patients with chronic fatigue syndrome
} T Siessmeier, W A Nix, J Hardt, M Schreckenberger, U T Egle, P Bartenstein

See end of article for authors' affiliations

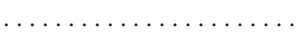

Correspondence to: Dr Thomas Siessmeier, Department of Nuclear Medicine, Johannes Gutenberg University, Langenbeckstr 1, 55101

Mainz, Germany; siessmeier@

nuklear.klinik.uni-mainz.de

Received

4 November 2002

In revised form 26

February

Accepted

28 February 2003

\begin{abstract}
Objectives: To evaluate cerebral glucose metabolism, assessed by 18-fluorodeoxyglucose positron emission tomography (FDG-PET), in patients with chronic fatigue syndrome (CFS), using an observer independent analytical approach; and to characterise any observed alterations by correlating them with neuropsychological deficits.

Methods: 26 patients (13 female, 13 male) were examined. They all fulfilled the CDC diagnostic criteria for CFS. Their ages ranged from 26 to 61 years (mean (SD) age, 43 (9.3) years). They underwent extensive psychometric testing including the hospital anxiety and depression scale (HADS) and the short form 36 item health questionnaire (SF-36). Brain FDG-PET was done in all the subjects. After stereotactic normalisation, single subject comparisons with an age and sex matched normal database $(n=18)$ and a group comparison between the patients and normal controls were undertaken, along with additional correlation analyses between brain metabolism and psychometric test scores.

Results: 12 of the 26 patients showed no significant decrease in FDG uptake compared with the controls. Of the remaining 14, 12 showed hypometabolism bilaterally in the cingulate gyrus and the adjacent mesial cortical areas. Five of these 12 patients also had decreased metabolism in the orbitofrontal cortex. The two remaining patients had hypometabolism in the cuneus/praecuneus. Correlation analyses showed significant correlations between some test scores (anxiety, depression, health related quality of life) but not fatigue and regional reductions in glucose metabolism.

Conclusions: Although abnormalities in FDG-PET were only detectable in approximately half the CFS patients examined, and no specific pattern for CFS could be identified, PET may provide valuable information in helping to separate CFS patients into subpopulations with and without apparent alterations in the central nervous system.
\end{abstract}

A lthough chronic fatigue syndrome (CFS) is not recognised as an independent syndrome by most neurologists and psychiatrists, ${ }^{1}$ this symptom complex is of enormous relevance for health economy. Estimations by the Centers of Disease Control (CDC) suggest that about 200 persons per 100000 are affected. This would mean that in the USA alone nearly 500000 people suffer from symptoms that fulfil the criteria for CFS. ${ }^{2}$

According to the diagnostic criteria established by the CDC, CFS is characterised by a sudden onset of debilitating, persistent fatigue and loss of energy which lasts for more than six months and cannot be attributed to other medical or psychiatric conditions. Particular subjective complaints of patients are headache, sore throat, tender and painful lymph nodes, muscle and joint pain, and substantial impairment of short term memory and concentration. ${ }^{34}$ Depression and related symptoms are common comorbidities in CFS, and there are studies indicating that major depressive disorder is more prevalent in CFS than in other chronic medical illnesses. ${ }^{5}$ The aetiology of CFS remains unclear, although abnormalities in serological, immunological, and central nervous system function have been reported. ${ }^{67}$

Up to now there have been no specific biomedical markers for the diagnosis of this disease. The diagnosis is exclusively based on patients' complaints and the fulfilment of a clinical case definition. ${ }^{78}$ Objective findings are lacking.

Functional neuroimaging, especially single photon emission computed tomography (SPECT) of regional cerebral blood flow, has been used to assess specific patterns of blood flow alteration in patients with CFS, but conflicting results have been obtained. Although most studies describe lowered regional cerebral blood flow in a variety of brain regions (mainly frontal and temporal), no consistency of findings can be observed across studies. ${ }^{\text {? }}$
A positron emission tomography (PET) study by Tirelli et al examined cerebral glucose metabolism using ${ }^{18} \mathrm{~F}$-fluorodeoxyglucose (FDG) and a region of interest based approach for data analysis. ${ }^{10}$ These investigators found significant hypometabolism compared with normal controls in the right mediofrontal cortex and the brain stem. They also compared CFS with depression and found hypometabolism bilaterally in the upper frontal regions in the latter.

So far there have been no PET studies using observer independent analytical approaches to determine regional changes in cerebral metabolism induced by CFS on a pixelwise basis. Furthermore, no attempts have been made to use PET or SPECT to correlate the neuropsychological alterations observed in CFS with regional impairments of glucose utilisation or regional cerebral blood flow.

Our aim in the present study was therefore to identify in an observer independent way individual alterations of glucose metabolism in a carefully selected population of CFS patients, in order to assess how often significant abnormalities occur among such patients and whether the abnormalities follow a specific pattern. In addition, we undertook a group analysis to correlate regional functional impairment with different neuropsychological alterations occurring in CFS patients.

Abbreviations: CDC, Centers for Disease Control; CFS, chronic fatigue syndrome; FDG-PET, 18-fluorodeoxyglucose positron emission tomography; FWHM, full width half maximum; HADS, hospital anxiety and depression scale; $\mathrm{HRQOL}$, health related quality of life; PET, positron emission tomography; SF-36, short form 36 item questionnaire; SCID, structured clinical interview for DSM-IV; SPECT, single photon emission computed tomography 


\section{METHODS \\ Patients}

A sample of 26 adults (mean (SD) age, 43 (9.3) years, range 26 to $61 ; 13$ male; 13 female) was drawn from 74 consecutive referrals for the evaluation of CFS seen at the University Hospital in Mainz. The 1992 CDC working case definition, ${ }^{8}$ together with the CDC exclusionary conditions and criteria for subgrouping variables, ${ }^{7}$ was used by a neurologist (WAN) to diagnose CFS. All patients with presumptive CFS were admitted to hospital for one week, during which they underwent an extensive physical, electrophysiological, and psychological examination (including all standard blood tests, microbiology, a variety of neurological examinations, and various psychometric tests such as the "symptom checklist 90 revised" and other measures of somatic and psychological complaints). The fatigue scale, the hospital anxiety and depression scale (HADS), and the short form 36 item questionnaire (SF-36) were chosen for the analyses because they assess distinct aspects of neuropsychological function with minimal overlap. Patients who were found to have an organic disease (two with diabetes, one with gluten induced enteropathy, and one with multiple sclerosis) or a psychotic disorder (two cases) that explained the fatigue were excluded. ${ }^{7}$ Major depressive disorder according to the Diagnostic and Statistical Manual of Mental Disorders (DSM-IV) criteria was also a reason for exclusion. Recent morphological imaging (computed tomography (CT)/magnetic resonance imaging (MRI) (done within six months of the study) did not reveal substantial deficits or relevant atrophy in this sample.

Eagerness to participate in the PET study among the patients initially selected was low because they were worried about radioactivity, so in the end it was only possible to study 26 individuals. All these patients gave written informed consent after the PET study protocol was explained to them. For controls we referred to a normal database consisting of 18 volunteers (mean (SD) age, 38 (11.5) years, range 21 to 55; 12 male, six female). Neurological or psychiatric diseases were excluded in the normal controls by physical examination and extensive neuropsychological testing (SCID). Although the mean age of the control population was slightly lower, this difference was not significant $(\mathrm{p}>0.05)$. The same was true for the balance between male and female subjects.

\section{Psychometric tests}

Before the PET scan was done, the patients completed a series of psychometric tests. The testing procedure took about 10 to 15 minutes. It contained, among others, a fatigue impairment scale, anxiety and depression scales, and a generic health related quality of life questionnaire. The fatigue scale used is the German version of a disability index originally developed by Buchwald and coworkers, ${ }^{11}$ comprising 16 visual analogue scales that refer to impairment in different domains of daily life (for example, household, job, leisure, partner) as a result of CFS. The construction of the scales is oriented towards the pain disability index, ${ }^{12}$ a widely used and validated measure. A single dimension was extracted through factor analysis (principal components analysis), which yielded a Cronbach's $\alpha$ of 0.94 in the present sample. High values represent severe impairment.

Anxiety and depression were assessed using the hospital anxiety and depression scale (HADS-D). ${ }^{13}{ }^{14}$ The HADS is a widely used questionnaire containing seven items for each dimension and having good reliability (Cronbach's $\alpha=0.81$ and 0.80 , respectively). This questionnaire was chosen because its items were designed particularly to avoid any questions relating to somatic complaints, which may be difficult to assess in the context of CFS. High values represent high levels of anxiety or depression.

The SF-36 (short form) is a self rating questionnaire designed to assess generic health related quality of life (HRQOL). ${ }^{15}{ }^{16}$ It was derived from a much longer functional status measure used in the medical outcomes study to facilitate the assessment of health related quality of life in clinical and research settings. The SF-36 consists of eight subscales: physical functioning, role limitations caused by physical problems, bodily pain, general health perceptions, vitality, social functioning, role limitations caused by emotional problems, and emotional wellbeing. Raw values of the SF-36 were transformed into $z$ scores based on population means and standard deviations, adjusted for age and sex, as described in the SF-36 manual (for details, see Hardt et al ${ }^{17}$ ). The eight domains were combined into two dimensions of health related quality of life-mental and physical-using a method described by Hays et al. ${ }^{18}$ High values represent good health related quality of life.

\section{PET scanning}

All PET scans were done at the department of nuclear medicine of the University Hospital Mainz on a Siemens ECAT EXACT PET scanner (Siemens Co, Erlangen, Germany). Psychotropic drug treatment, which was taken only by a minority of the patients, was discontinued before the PET for a period determined by the plasma half life of the drug. All studies were done under identical standardised resting conditions with closed eyes and in dim ambient light.

The patients were placed on the PET scanner bed with the head parallel to the canto-meatal line, using a laser device. After a 10 minute transmission scan with a ${ }^{68} \mathrm{Ga} / \mathrm{Ge}$ ring source to allow for proper attenuation correction, 330 to $410 \mathrm{MBq}$ of ${ }^{18}$ fluoro-2-deoxy-D-glucose (FDG) were injected into an antecubital vein. Thirty minutes later, a 30 minute emission scan in cross sectional mode consisting of three frames of 10 minutes' duration was acquired. Postprocessing included adding the three frames to a single frame. The data on the control population were acquired in an identical fashion. The PET camera has a field of view of $16 \mathrm{~cm}$ and a resolution of $6 \mathrm{~mm}$ full width half maximum (FWHM). Correction for dead time, scatter, and random coincidence was applied, and the images were reconstructed using filtered back projection with a $4 \mathrm{~mm}$ Hamming filter, resulting in images consisting of 47 slices with a slice thickness of $3.4 \mathrm{~mm}$ and a pixel size of $2 \mathrm{~mm}$ in a $128 \times 128$ matrix.

\section{Image analysis}

Image analysis was done two parts: the identification of patterns of abnormal glucose metabolism in individual patients, and group analysis to correlate regional impairment of glucose metabolism with various psychometric indices.

\section{Patterns of abnormal glucose metabolism in individual patients}

Individual comparisons with the normal controls were undertaken with an automated program (3D-SSP, Neurostat, University of Michigan, USA). This software package was chosen because it is dedicated to single subject analysis and has been extensively evaluated in various disorders, including dementia and epilepsy. ${ }^{19-26}$

The analysis started with stereotactic normalisation to the standard brain atlas of Talairach and Tournoux, ${ }^{19}$ which was done on a SUN workstation (SUN Microsystems Inc, Santa Clara, California, USA). In a second step, standardised individual three dimensional surface projections were generated $^{25}$ and related to the thalamus as the reference region. That region was chosen because even at very low significance thresholds we did not detect any differences between the groups in thalamic activity $(\mathrm{p}<0.05$ not corrected for multiple comparison). We therefore felt justified in using the thalamus as the most robust reference region in our patient sample. The reliability of this reference region has been demonstrated in previous evaluations. ${ }^{20} 21$ The surface projections were compared statistically on a pixelwise basis with the normal controls, resulting in parametric $z$ score images reflecting the standard deviation of the individual index brain compared with the normal controls. 


\begin{tabular}{|c|c|c|c|c|c|c|c|c|c|c|}
\hline \multirow[b]{2}{*}{$\begin{array}{l}\text { Patient } \\
\text { No }\end{array}$} & \multicolumn{2}{|c|}{ Demographics } & \multicolumn{5}{|c|}{ Psychometric test scores } & \multicolumn{3}{|c|}{ PET findings } \\
\hline & $\begin{array}{l}\text { Age } \\
\text { (years) }\end{array}$ & Sex & Anxiety & Depression & $\begin{array}{l}\text { HRQOL, } \\
\text { physical }\end{array}$ & $\begin{array}{l}\text { HRQOL, } \\
\text { mental }\end{array}$ & Fatigue & None & $\begin{array}{l}\text { Mesial } \\
\text { cortical }\end{array}$ & $\begin{array}{l}\text { Orbito- } \\
\text { frontal }\end{array}$ \\
\hline 1 & 49 & M & 13 & 11 & -2.4 & -2.4 & 72 & y & & \\
\hline 2 & 34 & $M$ & 10 & 7 & -3.0 & -2.6 & 46 & n & y & $\mathrm{n}$ \\
\hline 3 & 41 & $\mathrm{~F}$ & 11 & 10 & -2.3 & -1.5 & 66 & $n$ & y & $n$ \\
\hline 4 & 26 & $M$ & 8 & 7 & -2.2 & -1.4 & 34 & $\mathrm{n}$ & y & $n$ \\
\hline 5 & 35 & $\mathrm{~F}$ & 6 & 8 & -2.2 & -1.5 & 44 & y & & \\
\hline 6 & 34 & $M$ & 13 & 8 & -3.6 & -3.6 & 85 & n & $\mathrm{n}$ & y \\
\hline 7 & 40 & $F$ & 2 & 4 & -3.5 & -1.2 & * & y & & \\
\hline 8 & 43 & $M$ & 13 & 14 & -2.6 & -3.2 & 73 & y & & \\
\hline 9 & 43 & $M$ & 9 & 17 & -4.6 & -3.6 & 88 & y & & \\
\hline 10 & 54 & $\mathrm{~F}$ & 4 & 2 & -2.5 & -0.7 & 48 & $y$ & & \\
\hline 11 & 54 & $M$ & 7 & 12 & -2.1 & -0.5 & 83 & n & $y$ & y \\
\hline 12 & 52 & $\mathrm{~F}$ & 2 & 7 & -2.1 & -1.8 & 51 & $y$ & & \\
\hline 13 & 39 & $M$ & 17 & 8 & -1.9 & -3.3 & 42 & y & & \\
\hline 14 & 57 & $\mathrm{~F}$ & 3 & 10 & -2.3 & -1.5 & 87 & y & & \\
\hline 15 & 57 & $\mathrm{~F}$ & 7 & 4 & -1.2 & -1.6 & 31 & y & & \\
\hline 16 & 61 & $\mathrm{~F}$ & 7 & 10 & -1.7 & -1.2 & 63 & $\mathrm{n}$ & $y$ & $y$ \\
\hline 17 & 35 & $\mathrm{~F}$ & 3 & 11 & -1.6 & -1.0 & 76 & $y$ & & \\
\hline 18 & 42 & $M$ & 7 & 8 & -2.2 & -2.1 & 51 & $\mathrm{n}$ & $y$ & $\mathrm{n}$ \\
\hline 19 & 27 & $M$ & 10 & 3 & -0.7 & -0.7 & * & $n$ & cuneus & $n$ \\
\hline 20 & 48 & $\mathrm{~F}$ & 4 & 4 & -1.3 & -0.1 & 58 & $\mathrm{n}$ & cuneus & $\mathrm{n}$ \\
\hline 21 & 50 & $\mathrm{~F}$ & 14 & 7 & -2.9 & -2.9 & 69 & $\mathrm{n}$ & $\mathrm{n}$ & $y$ \\
\hline 22 & 41 & $M$ & 10 & 12 & -2.0 & -2.7 & 72 & $n$ & $y$ & y \\
\hline 23 & 47 & $M$ & 6 & 12 & -3.7 & -2.5 & 78 & $y$ & & \\
\hline 24 & 29 & $M$ & 6 & 7 & -4.4 & -1.2 & 80 & $\mathrm{n}$ & $y$ & $\mathrm{n}$ \\
\hline 25 & 47 & $\mathrm{~F}$ & 2 & 8 & -3.1 & -1.4 & 67 & $\mathrm{n}$ & y & $\mathrm{n}$ \\
\hline 26 & 41 & $F$ & 3 & 1 & -1.7 & 0.0 & 19 & $n$ & $y$ & $\mathrm{n}$ \\
\hline Mean & 43 & & 7.6 & 8.2 & -2.5 & -1.8 & 61.8 & & & \\
\hline SD & 9.3 & & 4.2 & 3.8 & 0.9 & 1.0 & 19.4 & & & \\
\hline
\end{tabular}

${ }^{*}$ Missing data.

$F$, female; HRQOL, health related quality of life (short form 36); $M$, male; $n$, no; PET, positron emission tomography; $y$, yes.

A relevant decrease in glucose metabolism was assumed if more than 50 adjacent pixels showed a value of $>2$ in the resulting $z$ score image. ${ }^{20}$ As this analysis requires no user interaction or a priori hypothesis, it can be regarded as observer independent.

An additional analysis based on proportional normalisation to the global mean was also undertaken. This analysis yielded similar results. Because some patients showed extensive cortical abnormalities, we felt that normalisation based on cortical activity was not adequate. Therefore these results are not reported here in detail.

\section{Correlation of glucose metabolism with psychometric indices}

In a second analytical step we aimed to identify in a group analysis areas where regional impairment of glucose metabolism was correlated with various psychometric indices. This was done using the SPM 99 software package (Wellcome Department of Cognitive Neurology, London, UK). ${ }^{27}$ Following stereotactic normalisation, this analysis started with a categorical group comparison between the patients in the CFS group $(n=26)$ and the normal controls $(n=18)$. This was done in order to identify the areas where glucose metabolism was most consistently impaired. Based on the entire CFS study population, we carried out correlation analyses (Spearman correlation, Fischer transformation) of regional glucose metabolism with the individual psychometric test scores measuring depression and anxiety (HADS), fatigue, and HRQOL. These analyses were restricted to voxels where the categorical comparison revealed differences $(\mathrm{p}<0.05$, uncorrected).$^{28}$

As it was the aim of this study to identify impairments that are specific for CFS in a sensitive manner, a generous signifi- cance threshold was applied ( $\mathrm{p}<0.001$; uncorrected) for all correlation analyses. ${ }^{29}$

\section{RESULTS \\ Psychometric tests}

The mean impairment resulting from CFS on the visual analogue scales was about 62 . This corresponds to more than the $50 \%$ impairment required for the definition of CFS. Reported anxiety (HADS) was in the normal range, but values for depression were increased. ${ }^{13}$ There were negative $z$ scores for HRQOL, implying a major decrease in the quality of life compared with the general population-that is, $2.45 \mathrm{SD}$ below normal in the physical and 1.77 SD below normal in the mental components. As all psychometric tests showed a meaningful range in the present sample, their use in a correlational analysis was reasonable. A summary is given in table 1 .

\section{PET data}

Individual comparisons with the normal database

Twelve of the 26 CFS patients (46\%) showed no significant abnormalities in comparison with the healthy control group. In 12 of the 14 remaining patients we identified areas with decreased glucose metabolism in the cingulate gyrus and adjacent mesial cortical areas (Brodmann areas 32, 24, 31, and 23). Five of these 12 patients showed additional decreases in the orbitofrontal/frontobasal cortex (Brodmann area 11). Figure 1 presents the patient (No 22 in table 1) with the most pronounced abnormalities. This 41 year old man was one of the most severely affected. During the course of the disease he developed severe anxiety and an obsessive-compulsive disorder. The remaining two patients (mother and son) showed a different pattern of decreased glucose metabolism centred in 


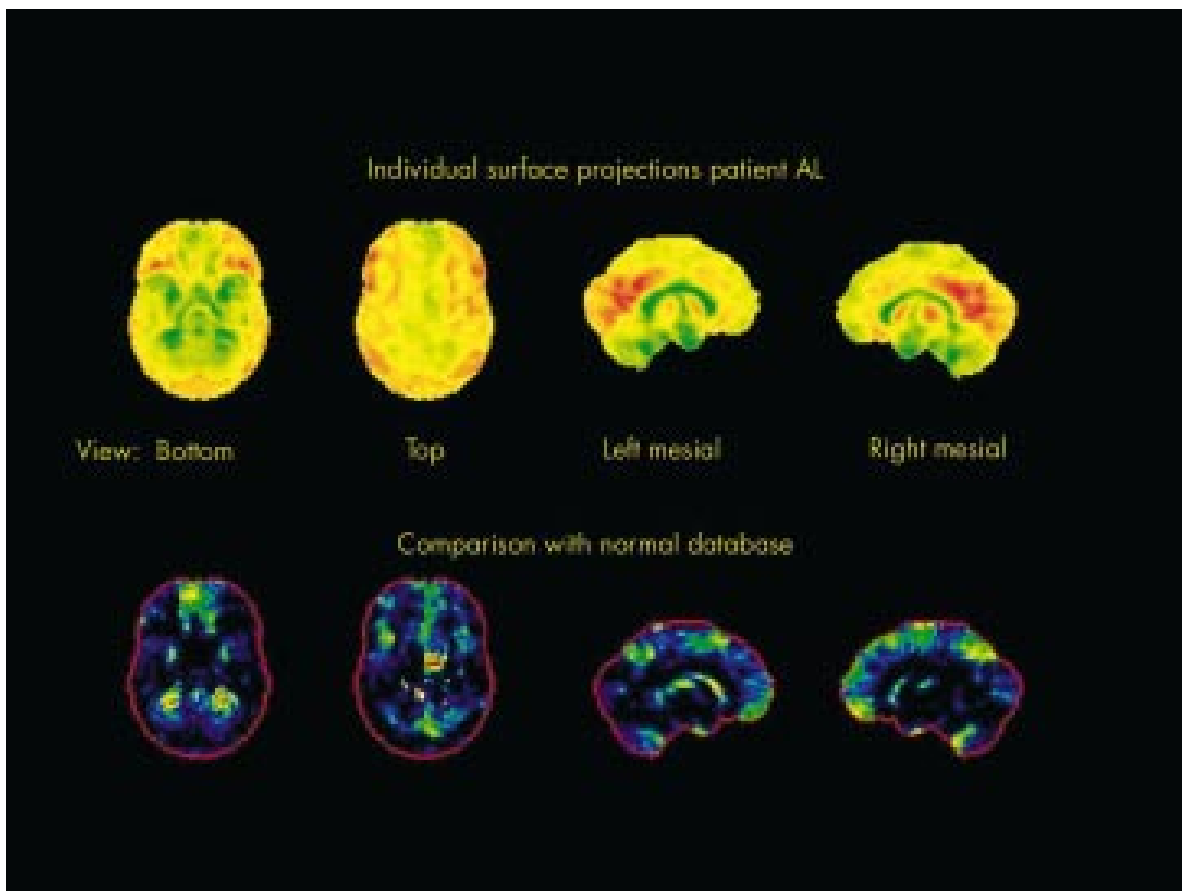

Figure 1 Surface projections and comparison with normal controls of the patient with the most pronounced abnormalities (decreases) in the FDG-PET image. FDG-PET, fluorodeoxyglucose positron emission tomography.

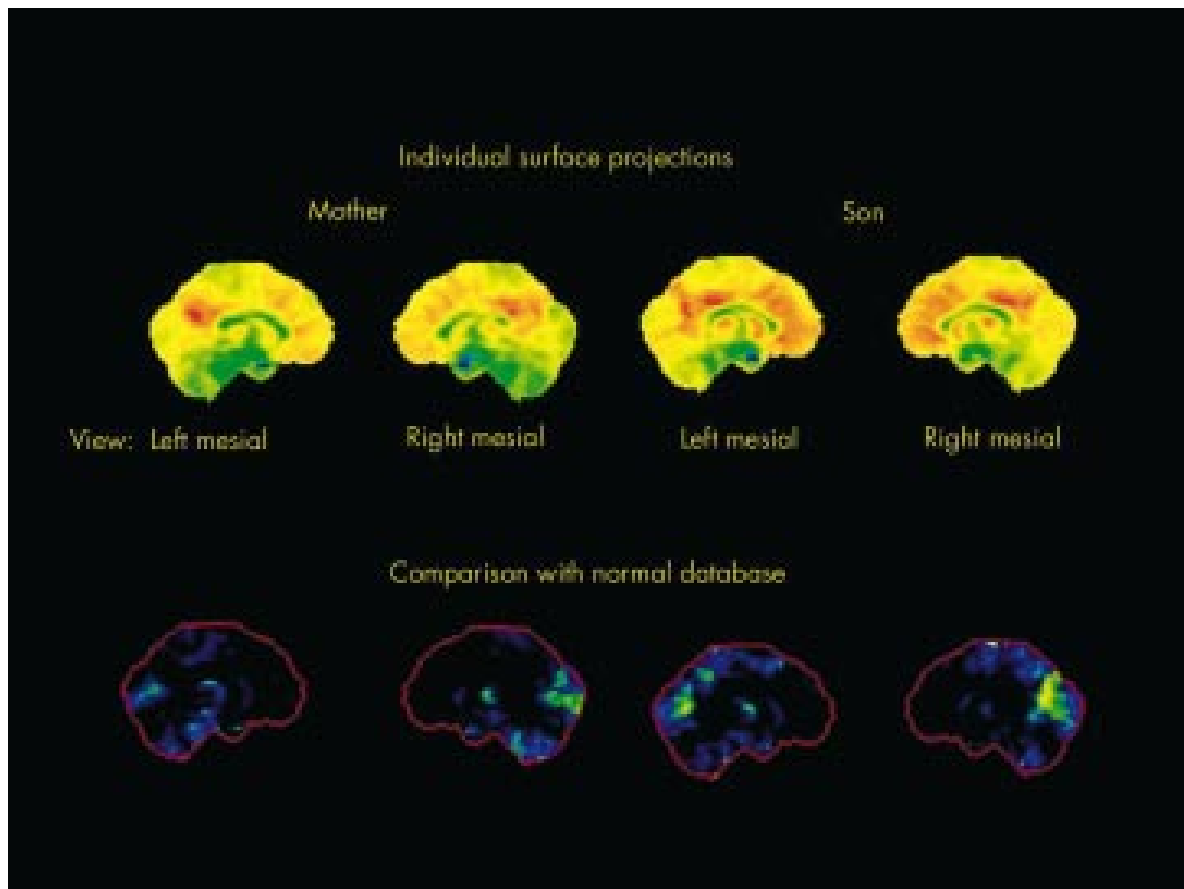

Figure 2 Surface projections and comparison with normal controls of the patients (mother and son) with decreases located in the cuneus/precuneus region.

the cuneus/precuneus region. Remarkably, these two patients had no abnormalities in the cingulate and orbitofrontal regions (fig 2).

Table 1 summarises the findings of the individual comparison of all 26 patients including demographic data.

\section{Group comparison}

The categorical comparison between the patient group and the 18 healthy controls showed areas of significantly decreased glucose metabolism only in the orbitofrontal cortex (15\% decrease in the maximum pixel of this cluster $)(p<0.001)$. When a more generous threshold was applied $(p<0.05)$ the cingulate gyrus and the adjacent mesial cortical areas showed a decrease in glucose metabolism as well ( $8 \%$ in the maximum pixel of this cluster). These findings are in good agreement with the individual analyses.

\section{Correlation analyses}

The pixelwise correlation analyses between the decrease in glucose metabolism and the psychometric testing scales 


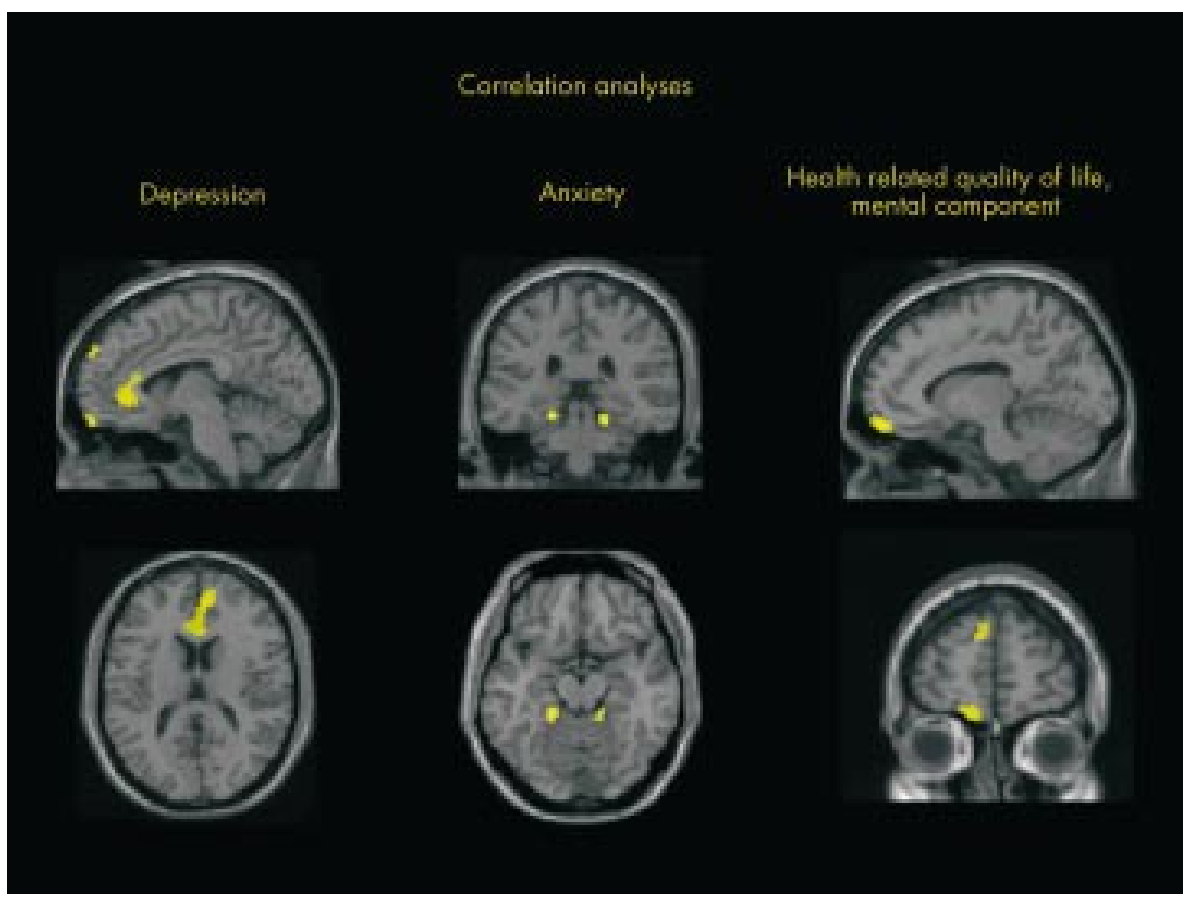

Figure 3 Results of the correlation analyses with SPM 99 software package $(n=26)$. The significant voxels are projected on standardised magnetic resonance imaging in transaxial, coronal, and sagittal views. For illustrative purposes, a significance threshold of $p<0.005$ was chosen.

\begin{tabular}{|c|c|c|c|c|c|c|}
\hline \multirow{2}{*}{$\begin{array}{l}\text { Psychometric } \\
\text { test }\end{array}$} & \multirow[b]{2}{*}{ Area } & \multirow{2}{*}{$\begin{array}{l}\text { Brodmann } \\
\text { area }\end{array}$} & \multicolumn{3}{|c|}{$\begin{array}{l}\text { Talairach coordinates } \\
(\mathrm{mm})\end{array}$} & \multirow[b]{2}{*}{ z Score } \\
\hline & & & $x$ & y & z & \\
\hline \multirow{3}{*}{$\begin{array}{l}\text { Depression } \\
\text { (HADS) }\end{array}$} & Anterior cingulate gyrus, left & 24 & -10 & 36 & -4 & 3.84 \\
\hline & Superior frontal gyrus, right & 6 & 26 & 20 & 60 & 3.53 \\
\hline & Medial frontal gyrus, right & 10 & 10 & 50 & 16 & 3.36 \\
\hline \multirow{2}{*}{$\begin{array}{l}\text { Anxiety } \\
\text { (HADS) }\end{array}$} & Parahippocampal gyrus, right & 36 & 20 & -20 & -22 & 4.36 \\
\hline & Parahippocampal gyrus, left & 36 & -20 & -26 & -18 & 4.32 \\
\hline \multirow{3}{*}{$\begin{array}{l}\mathrm{HRQOL} \\
\text { mental }\end{array}$} & Medial frontal gyrus, left & 11 & -10 & 60 & -18 & 4.27 \\
\hline & Temporal inferior gyrus/uncus, left & $20 / 36$ & -20 & 6 & -32 & 3.89 \\
\hline & Parahippocampal gyrus, right & 35 & 18 & -30 & -6 & 3.84 \\
\hline
\end{tabular}

showed the following results in the CFS patient group as a whole $(\mathrm{n}=26)$.

\section{HADS depression scale}

We found correlations between the severity of depression and changes in the mesial orbitofrontal cortex on the right, the right temporal superior and medial gyrus, and the anterior cingulate gyrus, at $z$ score levels of $>3.10(\mathrm{p}<0.001)$ (fig 3 , table2).

\section{HADS anxiety scale}

Anxiety was exclusively correlated with a bilateral decrease in glucose metabolism at the border between the mesial temporal cortex and the cerebellum. As the maximum significant pixel on both sides was located in the parahippocampal gyrus it seems reasonable to attribute the correlations to this structure (fig 3, table2).

\section{Fatigue scale}

Remarkably no correlation between regional decreases in glucose metabolism and the severity of perceived fatigue was observed.

\section{Health related quality of life}

Impairment in the mental component of HRQOL was very closely correlated with decreased glucose metabolism in the orbitofrontal region, mainly on the left side $(z=4.3$; $\mathrm{p}<0.0001$ ). In addition, other areas with a less tight correlation were located in the mesial and dorsolateral prefrontal regions and in the mesial and superior temporal cortex. In contrast to this, no correlation between the physical component of HRQOL and regional impairment of glucose metabolism was found.

Table 2 gives an overview of the significant correlations between the psychometric test results and the regional decreases in glucose metabolism that were found. 


\section{DISCUSSION}

The main finding of the individual comparisons in our study is that, in the patients fulfilling the diagnostic criteria for CFS, only about half the cases showed abnormalities of glucose metabolism detectable using an observer independent approach. In most of the abnormal cases the abnormalities were focused on the anterior cingulate gyrus/mesial frontal cortex and the orbitofrontal cortex. Two patients (relatives) showed a very distinct pattern, with decreased glucose metabolism centred on the cuneus/precuneus region.

As no consistent pattern of metabolic abnormality was detectable in this patient sample, this individual observer independent analysis does not support the assumption that CFS is a homogeneous disease entity. ${ }^{30} 31$

The most homogeneous pattern detectable in our sample is in line with the results of the SPECT study on CFS by Schwartz et al..$^{32}$ The observed results are also comparable with the findings of various other SPECT studies where significantly lowered regional cerebral blood flow was found in a several brain regions-mainly in the lateral frontal, lateral temporal, and medial temporal lobes-in CFS patients compared with normal controls (for a review, see Mayberg ${ }^{9}$ ). Additional abnormalities in the brain stem described by Costa et al in a pilot study using region of interest analysis ${ }^{33}$ were not detectable in our patient sample. Even an exploratory analysis in our group comparison at a very low significance threshold $(\mathrm{p}<0.05$, not corrected) did not reveal significant changes. Owing to the stereotactic normalisation approach necessary for the pixelwise analyses in our study, subtle changes in small structures like the brain stem might not be detectable with the same sensitivity. On the other hand region of interest based analyses in this area are very sensitive to small changes in region size and location. Thalamic hyperperfusion in CFS, as described by MacHale et al, ${ }^{34}$ was also not apparent in our group analysis, even at a very low significance threshold ( $p<0.05$ not corrected for multiple comparisons). This might reflect differences in the methodology used (FDG-PET $v$ regional cerebral blood flow SPECT) and patient selection (depressed patients were excluded by MacHale et al).

Correlation analyses revealed region specific impairment of glucose metabolism for depression, anxiety, and the mental component of HRQOL, but not for the psychophysical abnormalities, reflecting the CDC diagnostic criteria for CFS (fatigue und impairment in the physical component of health related quality of life).

The location of the area of decreased metabolism correlating with depression in our CFS patients has striking similarities to the region (the most anterior parts of the cingulate gyrus) described by Drevets as showing decreased blood flow and metabolism in patients with unipolar and bipolar depression..$^{35}$ In line with these observations is the fact that the patterns detected in the majority of abnormal patients in our sample by individual comparison share similarities with results of PET and SPECT imaging in major depression. ${ }^{36}$ Resting state studies on patients with unipolar depression consistently describe hypofrontality, mainly in the dorsolateral prefrontal cortex and the anterior cingulate..$^{35-41}$ This suggests that depression is one of the key factors inducing abnormalities of neuronal function in some CFS patients, detectable with functional imaging. The relation of the impairments in our CFS group to depression is also supported by findings of Tashiro et al, ${ }^{42}$ who described reductions in cerebral metabolism in cancer patients in similar areas (especially the anterior cingulate and dorsolateral prefrontal cortex) and related these to the degree of depression in these patients. Comparable changes in the frontal lobe have also been reported in patients with rheumatoid arthritis. ${ }^{43}$ However, attributing hypometabolism in anterior cingulate and frontal cortex in CFS patients to depression does not help to determine whether depression is a triggering factor for CFS or the result of chronic suffering from fatigue.
Additional dimensions of neuropsychological impairment in CFS patients were correlated with decreased cerebral glucose metabolism in other locations. Thus the severity of anxiety was mainly correlated with changes in glucose metabolism in the mesial temporal structures including the amygdalo-hippocampal complex. This area appears to be a key structure in a circuit that governs negative affect. The amygdala has been shown in animal experiments to be necessary for the development of conditioned fear and is said to be important in the expression of anxiety disorders in humans ${ }^{44-47}$ Anxiety as a subcomponent of the CFS syndrome complex may make a major contribution to mesiotemporal changes observable with neurofunctional imaging.

A third area correlated mainly with subjective impairment in the quality of life was the orbitofrontal cortex. This area, as an element of the paralimbic belt, has also been found to be involved in various psychiatric disorders including anxiety, obsessive-compulsive disorder, addiction, and physiological states such as aversion. ${ }^{48-50}$ As perception of quality of life is clearly dependent on whether emotions experienced are rated as mainly positive or mainly negative, a correlation between subjective quality of life and the orbitofrontal cortex seems appropriate. It has been shown in an international comparison that severe impairment of quality of life is a common finding in CFS patients. ${ }^{17}$ This is important, as our findings with respect to this impairment can be generalised and do not seem to be restricted to the specific patients or location under study. Dysfunction of the orbitofrontal cortex can also result from chronic stress experiences during childhood or later in life..$^{52}$ This correlation was confirmed in an animal study, ${ }^{53}$ as well as in PET studies in patients with torture related post-traumatic stress disorder ${ }^{54}$ and in previously institutionalised Romanian orphans. ${ }^{55}$

In contrast to these clear correlations, it is remarkable that objective impairment of quality of life and the severity of fatigue-both of which are CDC diagnostic criteria and were therefore present in all our CFS patients-did not have a metabolic correlate. Abnormalities of glucose metabolism in CFS thus do not seem to be specific for the syndrome but rather are correlated with different neuropsychological symptoms shown during the course of the disease. They can be observed in other clearly defined disorders with similar symptoms, so they may be an expression of the same mediating neuroanatomy underlying these symptoms rather than the reflection of neuronal lesions caused by CFS. Differences in the level of symptom expression could be responsible for the inconsistency of the results of previous PET/SPECT studies on CFS patients. However, it seems that disease severity is determined not by the level of symptom expression but rather by the symptom mix and by the number of individual symptoms. Our data therefore support the hypothesis that the CFS case definition does not define a single disease entity but rather recruits patients with different health problems but similar subjective complaints. ${ }^{29} 30$

\section{Conclusions}

Although abnormalities in FDG-PET were only detectable in approximately half the CFS patients examined and no pattern specific for CFS could be determined, PET may provide valuable information in helping to separate patients with this condition into subpopulations with and without apparent alterations of the central nervous system.

\section{Authors' affiliations}

T Siessmeier, M Schreckenberger, P Bartenstein, Department of Nuclear Medicine, Johannes Gutenberg University, Mainz, Germany W A Nix, Department of Neurology, Johannes Gutenberg University, Mainz

J Hardt, U T Egle, Department of Psychosomatic Medicine and

Psychotherapy, Johannes Gutenberg University, Mainz

Competing interests: none declared 


\section{REFERENCES}

1 Wessely S. Chronic fatigue syndrome. J Neurol Neurosurg Psychiatry 1991;54:669-71.

2 Steele L, Dobbins JG, Fukuda K, et al. The epidemiology of chronic fatigue in San Francisco. Am J Med 1998;105:83-90S.

3 Holmes GP, Kaplan JE, Gantz NM, et al. Chronic fatigue syndrome: a working case definition. Ann Intern Med 1988;108:387-9.

4 Komaroff AL, Buchwald D. Symptoms and signs of chronic fatigue syndrome. Rev Infect Dis 1991;13(suppl 1):S8-11.

5 Kruesi MJ, Dale J, Straus SE. Psychiatric diagnoses in patients who have chronic fatigue syndrome. J Clin Psychiatry 1989;50:53-6.

6 Bell DS. Chronic fatigue syndrome update. Findings now point to CNS involvement. Postgrad Med 1994:96:73-6, 79-81.

7 Fukuda K, Straus SE, Hickie I, et al. The chronic fatigue syndrome: a comprehensive approach to its definition and study. International Chronic Fatigue Syndrome Study Group. Ann Intern Med 1994;121:953-9.

8 Schluederberg A, Straus SE, Peterson P, et al. NIH conference. Chronic fatigue syndrome research. Definition and medical outcome assessment. Ann Intern Med 1992;117:325-31.

9 Mayberg H. Functional neuroimaging in CFS: applications and limitations. J Chron Fatigue Syndr 1995;1:9-20.

10 Tirelli U, Chierichetti $F$, Tavio $M$, et al. Brain positron emission tomography (PET) in chronic fatigue syndrome: preliminary data. Am $J$ Med 1998;105:54-8S

11 Herrell R, Goldberg J, Hartman S, et al. Chronic fatigue and chronic fatigue syndrome: a co-twin control study of functional status. Qual Life Res 2002;11:463-71

12 Tait RC, Chibnall JT, Krause S. The Pain Disability Index: psychometric properties. Pain 1990;40:171-82.

13 Zigmond AS, Snaith RP. The hospital anxiety and depression scale. Acta Psychiatr Scand 1983;67:361-70.

14 Herrmann C, Buss U, Snaith RP. HADS-D hospital anxiety and depression scale - Deutsche version. Bern: Huber, 1995.

15 Ware JE, Snow KK, Kosinski M, et al. SF-36 health survey: manual and interpretation guide. Boston: New England Medical Center, The Health Institute, 1993

16 Bullinger M, Kirchberger I. SF-36 Fragebogen zum Gesundheitszustand. Göttingen: Hogrefe, 1998

17 Hardt J, Buchwald D, Wilks D, et al. Health-related quality of life in patients with chronic fatigue syndrome. An international study. $J$ Psychosom Res 2001;51:431-4.

18 Hays RD, Alonso J, Coons SJ. Possibilities for summarizing health-related quality of life when using a profile instrument. In: Staquet M, Hays RD, Fayers PM, eds. Quality of life assessment in clinical trials. Oxford: Oxford University Press, 1998:143-53

19 Talairach J, Tournoux P. Co-planar stereotaxic atlas of the human brain. New York: Thieme, 1988.

20 Drzezga A, Arnold S, Minoshima S, et al. ${ }^{18} \mathrm{~F}-\mathrm{FDG}$ PET studies in patients with extratemporal and temporal epilepsy: evaluation of an observer-independent analysis. J Nucl Med 1999;40:737-46.

21 Bartenstein P, Minoshima S, Hirsch C, et al. Quantitative assessment of cerebral blood flow in patients with Alzheimer's disease by single photon emission computed tomography. J Nucl Med 1997;38:1095-101.

22 Reiman EM, Caselli RJ, Yun LS, et al. Preclinical evidence of Alzheimer's disease in persons homozygous for the epsilon 4 allele for apolipoprotein E. N Engl J Med 1996;334:752-8.

23 Minoshima S, Koeppe RA, Mintun MA, et al. Automated detection of the intercommissural line for stereotactic localization of functional brain images. J Nucl Med 1993;34:322-9.

24 Minoshima S, Koeppe RA, Frey RA, et al. Anatomic standardization: linear scaling and nonlinear warping of functional brain images. J Nuc Med 1994:35:1528-37.

25 Minoshima S, Koeppe RA, Frey KA, et al. Stereotactic PET atlas of the human brain: aid for visual interpretation of functional brain images. $J$ Nucl Med 1994;35:949-54

26 Minoshima S, Frey KA, Koeppe RA, et al. A diagnostic approach in Alzheimer's disease using three-dimensional stereotactic surface projections of fluorine-18-FDG PET. J Nucl Med 1995;36:1238-48.

27 Friston KJ, Ashburner J, Poline JB, et al. Spatial registration and normalisation of images. Hum Brain Map 1995;2:165-89.

28 Bartenstein P, Weindl A, Spiegel S, et al. Central motor processing in Huntington's disease. A PET study. Brain 1997:120:1553-67.

29 Boecker H, Ceballos-Baumann A, Bartenstein P, et al. Sensory processing in Parkinson's and Huntington's disease: investigations with 3D H(2)(15)O-PET. Brain 1999; 122:1651-65.
30 Nix WA, Egle TU. Das chronische Erschöpfbarkeitssyndrom. Aktuelle Neurol 1998:25:6-12.

31 Wessely S, Nimnuan C, Sharpe M. Functional somatic syndromes: one or many? Lancet 1999;354:936-9.

32 Schwartz RB, Komaroff AL, Garada BM, et al. SPECT imaging of the brain: comparison of findings in patients with chronic fatigue syndrome, AIDS dementia complex, and major unipolar depression. Am J Roentgenol 1994;162:943-51

33 Costa DC, Tannock C, Brostoff J. Brainstem perfusion is impaired in chronic fatigue syndrome. QJM 1995;88:767-73.

34 MacHale SM, Lawrie SM, Cavanagh JT, et al. Cerebral perfusion in chronic fatigue syndrome and depression. Br J Psychiatry 2000; 176:550-6.

35 Drevets WC. Prefrontal cortical-amygdala metabolism in major depression. Ann NY Acad Sci 1999;877:614-37.

36 Videbech P. PET measurements of brain glucose metabolism and blood flow in major depressive disorder: a critical review. Acta Psychiatr Scand 2000;101:11-20.

37 Martinot JL, Hardy P, Feline A, et al. Left prefrontal glucose hypometabolism in the depressed state: a confirmation. Am J Psychiatry 1990;147:1313-17.

38 Dolan RJ, Friston KJ. Positron emission tomography in psychiatric and neuropsychiatric disorders. Semin Neurol 1989;9:330-7.

39 Baxter LR, Schwartz JM, Phelps ME, et al. Reduction of prefrontal cortex glucose metabolism common to three types of depression. Arch Gen Psychiatry 1989;46:243-50.

40 Hurwitz TA, Clark C, Murphy E, et al. Regional cerebral glucose metabolism in major depressive disorder. Can J Psychiatry 1990;35:684-8

41 Bench CJ, Friston KJ, Brown RG, et al. Regional cerebral blood flow in depression measured by positron emission tomography: the relationship with clinical dimensions. Psychol Med 1993;23:579-90.

42 Tashiro $M$, Juengling FD, Reinhardt M, et al. Depressive state and regional cerebral activity in cancer patients - a preliminary study. Med Sci Monit 2001;7:687-95.

43 Bartolini M, Candela M, Brugni $M$, et al. Are behaviour and motor performances of rheumatoid arthritis patients influenced by subclinical cognitive impairments? A clinical and neuroimaging study. Clin Exp Rheumatol 2002;20:491-7

44 Davidson RJ. Anxiety and affective style: role of prefrontal cortex and amygdala. Biol Psychiatry 2002;51:68-80

45 Aggleton JP. The contribution of the amygdala to normal and abnorma emotional states. Trends Neurosci 1993;16:328-33.

46 LeDoux J. The emotional brain: the mysterious underpinnings of emotional life. New York: Touchstone Press, 1996.

47 Saxena S, Brody AL, Maidment KM, et al. Localized orbitofrontal and subcortical metabolic changes and predictors of response to paroxetine treatment in obsessive-compulsive disorder. Neuropsychopharmacology 1999:21:683-93.

48 Rauch SL, Savage CR, Alpert NM, et al. The functional neuroanatomy of anxiety: a study of three disorders using positron emission tomography and symptom provocation. Biol Psychiatry 1997;42:446-52.

49 Small DM, Zatorre R, Dagher A, et al. Changes in brain activity related to eating chocolate: from pleasure to aversion. Brain 2001;124:172033.

50 Daglish MR, Weinstein A, Malizia AL, et al. Changes in regional cerebral blood flow elicited by craving memories in abstinent opiate-dependent subjects. Am J Psychiatry 2001;158:1680-6.

51 Kaufman J, Plotsky PM, Nemeroff CB, et al. Effects of early adverse experiences on brain structure and function: clinical implications. Biol Psychiatry 2000;48:778-90.

52 Davidson RJ, Jackson DC, Kalin NH. Emotion, plasticity, context, and regulation: perspectives from affective neuroscience. Psychol Bull 2000;126:890-909.

53 Arnsten AF. Stress impairs prefrontal cortical function in rats and monkeys: role of dopamine D1 and norepinephrine alpha-1 recepto mechanisms. Prog Brain Res 2000:126:183-92.

54 Fernandez $M$, Pissiota $A$, Frans $O$, et al. Brain function in a patient with torture related post-traumatic stress disorder before and after fluoxetine treatment: a positron emission tomography provocation study. Neurosci Lett $2001 ; 297: 101-4$

55 Chugani HT, Behen ME, Muzik O, et al. Local brain functional activity following early deprivation: a study of postinstitutionalized Romanian orphans. Neuroimage $2001 ; 14: 1290-301$ 